\title{
Tiempo Libre y Trabajo Doméstico en Su Relación con Daños Psicosociales en Docentes de una Universidad Pública Mexicana
}

\section{LEISURE TIME AND HOUSEHOLD WORK AND THEIR RELATIONSHIP TO PSYCHOSOCIAL HEALTH EFFECTS IN TEACHERS AT A MEXICAN PUBLIC UNIVERSITY}

\section{Susana Martínez Alcántara', Francisco López Barón², Omar Alejandro Garcia Contreras ${ }^{3}$}

1. Doctora en Ciencias de la Salud en el Trabajo. Maestra en Medicina Social y Licenciada en Psicología. Profesora-investigadora de la Maestría en Ciencias en Salud de los Trabajadores. Universidad Autónoma Metropolitana-Xochimilco. México, D.F.

2. Doctor en Ciencias de la Salud en el Trabajo. Máster en Clínica en Salud Mental. Licenciado en Psicología. Profesor de Ergonomía y Psicosociología Aplicada. Institut Universitari de Ciència i Tecnologia (IUCT). Mollet del Vallès. Barcelona. España.

3. Maestro en Psicología Social de Grupos e Instituciones. Licenciado en Psicología. Maestría en Ciencias en Salud de los Trabajadores, Universidad Autónoma Metropolitana-Xochimilco. México, D.F.

\begin{abstract}
RESUMEN
Objetivos: Estudio de la salud en el trabajo en docentes de la Universidad Autónoma Metropolitana-Xochimilco, considerando ciertas condiciones relacionadas con el uso del tiempo libre y el trabajo doméstico que pueden asociarse tanto positiva como negativamente con el bienestar y la salud. Material y métodos: Estudio observacional, transversal y analítico en una población académica. Se aplicó una encuesta individual a una población académica seleccionada aleatoriamente, participando 199 docentes universitarios, se exploraron las exigencias laborales, actividades domésticas y el uso del tiempo libre, así como daños en la salud. Resultados: Hay mayores exigencias laborales, tareas domésticas y trastornos en la mujer. Los daños a la salud muestran las siguientes tasas de prevalencia para mujeres y hombres: músculo-esqueléticos 51 vs 29; distrés 50 vs 35; Disfonía 48 vs 31 y trastorno del sueño 41 vs 25 , respectivamente, asociadas estadísticamente. Se identificaron mediante análisis de cluster efectos positivos a través de la cercanía estrecha de conglomerados que dieron cuenta del desarrollo de manera sostenida de actividades durante el tiempo libre y la ausencia de trastornos en la salud. Conclusiones: Se recomienda una mayor organización de las actividades laborales que permitan un uso más activo del tiempo libre, debiendo enfatizar la realización del ejercicio físico, que resultó ser el principal elemento protector contra trastornos en la salud física y mental.
\end{abstract}

(Martínez S, López F, García 0, 2013. Tiempo Libre y Trabajo Doméstico en su Relación con Daños Psicosociales en Docentes de una Universidad Pública Mexicana. Cienc Trab. Sep-Dic; 15 [48]: 165-172).

Palabras clave: TIEMPO LIBRE, TRABAJO DOMÉSTICO, DOCENTES UNIVERSITARIOS, TRASTORNOS MENTALES Y FÍSICOS.

\section{ABSTRACT}

Objectives: To conduct an occupational health study of teaching staff at the Universidad Autónoma Metropolitana-Xochimilco. The study considers conditions relating to use of leisure time and household work which can be associated both positively and negatively with well-being and health. Materials and methods: Observational, cross-sectional, analytical study of an academic population. A survey was applied to a single randomly selected academic population. The participants were 199 university teaching staff. The survey asked about work demands, household activities and the use of leisure time, and adverse health effects. Results: More household tasks and work demands, and more adverse health effects, were found in women. Prevalence rates for statistically associated adverse health effects in women and men respectively were: musculoskeletal disorders 51 vs 29; stress 50 vs 35; dysphonia 48 vs 31; and sleep disorders 41 vs 25 . Positive effects were identified by means of cluster analysis by the close proximity of clusters showing sustained activities carried out during leisure time and the absence of adverse health effects. Conclusions: Better organization of work activities to enable more active usage of leisure time is recommended, with an emphasis on physical activity, which is the main protective measure against harm to physical and mental health.

Key words: LEISURE TIME, HOUSEHOLD WORK, UNIVERSITY TEACHERS, ADVERSE MENTAL AND PHYSICAL HEALTH EFFECTS.
Correspondencia / Correspondence:

Susana Martínez Alcántara

Calzada del Hueso 1100, Col. Villa Quietud, Del. Coyoacán,

C.P. 04960, D. F. México.

e-mail smartin@correo.xoc.uam.mx

Tel.: +525554837205 • Fax: +525554837537

Recibido: 27 de Noviembre 2013 / Aceptado: 12 Diciembre 2013

\section{INTRODUCCIÓN}

Desde finales de la década de los 80 del siglo pasado, las políticas laborales que han venido definiendo los quehaceres macro-sectoriales de la población se vinculan con cambios dirigidos a sortear las graves crisis, especialmente esta última que se ha ido instaurando en los países tanto desarrollados como dependientes. Los cambios se han dado en términos: laborales, sociales, de salud, de educación, etc. Y se han traducido en políticas que han tenido repercusión a distintos niveles, aunque en términos concretos dan cuenta de un fenómeno conocido en el mundo del trabajo: la flexibilización laboral. Según Acevedo, esta “...actúa sobre la 
estabilidad del empleo, las formas y tiempos de contratación, los horarios de trabajo, la fijación de salarios, la seguridad en el trabajo y los ritmos e intensidad del trabajo, afectando en consecuencia la vida de los trabajadores". ${ }^{1}$

De manera paralela a este fenómeno, el incremento de la participación femenina en el mundo del trabajo remunerado se acentuó en las dos últimas décadas del siglo pasado ${ }^{2}$ lo que llevó a una reestructuración de los "roles" dentro de la familia tradicional ${ }^{3}$, además de producirse una serie de hechos que caracterizan la sociedad actual: aumento del número y tipología de familias reduciéndose el tamaño de las mismas; bajas tasas de fertilidad, incremento de la esperanza de vida, del número de divorcios que ha conllevado un aumento de familias monoparentales, madres asalariadas, etc.

En relación con las madres asalariadas, si bien no todos los gobiernos, empresas y/u organizaciones lo han tratado de la misma manera y, parece que, en la mayoría de casos no resuelven los obstáculos que encuentra la maternidad sin que la mujer deba renunciar a su desarrollo profesional ${ }^{4}$, teniendo en muchos casos que optar por la realización de una doble jornada laboral o por un empleo de tiempo parcial o marginal ${ }^{4}$, se observa que algunas organizaciones han establecido además de programas preventivos, la armonización de la vida laboral y personal a través de Programas de Ayuda al Empleado ${ }^{5}$. La participación en dichos programas son una elección personal. Ante el planteamiento de poder cambiar la jornada de tiempo completo por una a tiempo parcial $^{6}$, únicamente lo harían un 29\% de las mujeres, ya que ganarian menos dinero (68\%) y reduciría sus posibilidades de promoción (27\%). En el fondo de todo ello, la necesidad de permanecer en la empresa por parte del trabajador se debe principalmente a cuestiones económicas, sociales o psicológicas (necesidad de pertenencia, identidad laboral, etc.).

La mayoría de las mujeres, aun trabajando fuera del hogar de manera remunerada, continúan asumiendo la responsabilidad de las tareas consideradas socialmente como femeninas relacionadas con el cuidado del hogar, de los hijos y la organización de la vida familiar ${ }^{6}$, lo que ha dado pie a identificar una mayor frecuencia de trastornos mentales ${ }^{7}$ a medida que aumenta el número de hijos o diversas consecuencias sobre la calidad de vida de los docentes ${ }^{8}$ debido a la carga de trabajo y a la multiplicidad de tareas que se realizan, sobre todo en aquellos que han optado por participar en un programa de estímulos que permita mejorar los ingresos salariales. Esta situación alcanza el periodo de reposo, que teóricamente debería permitir la recuperación de las energías gastadas durante la actividad laboral.

El reposo se lleva a cabo en el tiempo libre, entendido como el periodo que le queda al individuo después de asumir sus obligaciones diarias (laborales, educativas, etc.). En este trabajo se distingue el tiempo libre de "no ocio" y en actividades de "ocio", siendo estas últimas cubiertas por aquellos momentos en que tras satisfacer las obligaciones, el individuo puede, más o menos, escoger libremente una actividad, sin un fin concreto. Su desarrollo debe resultarnos satisfactorio y placentero ${ }^{9}$, la condición del ocio es no tener la necesidad de estar ocupado. El ocio suele diferenciarse entre activo (ocio) y pasivo (ociosidad).

El tiempo libre es utilizado de acuerdo con lo que cada individuo considera más apropiado, que puede ir desde el compartir con la familia y/o amigos, el salir de paseo dentro o fuera del núcleo urbano o rural, el desarrollo de ejercicio físico, la participación comunitaria y/o político-sindical, el disfrute de actividades cultu- rales, la formación y/o actualización profesional de los miembros de la familia, hasta pasar la mayor parte de ese tiempo de forma pasiva ante la televisión.

Sin embargo, el tiempo libre ha venido reduciéndose cada vez más, debido a que la jornada laboral "no reglamentada" o "no oficial" se ha extendido hasta abarcar los fines de semana, los días festivos y de vacaciones, y parece ser esa la constante actual en el mundo del trabajo, ya que un estudio divulgado por la agencia DPA $^{10}$ indica que la mitad de los empleados alemanes no desconecta del trabajo en vacaciones. El 49\% de los mismos trabaja entre dos y tres horas más diarias; y un 10\% más de tres horas diarias. Dos tercios deben trabajar los fines de semana. En relación con las personas que trabajan en vacaciones, dicho estudio señala que son un 44\% de empleados en China, 27\% en la India, 21\% en Japón y un 17\% en Estados Unidos (USA) convirtiéndose casi en "norma” trabajar en fin de semana.

Esta reducción del tiempo alcanza tanto a hombres como a mujeres, lo que ha llevado a una mayor participación del género masculino en diversas tareas relacionadas con el hogar y con el cuidado de los hijos. Este es un fenómeno correlacionado con una mayor participación de las mujeres en el mercado laboral ${ }^{3}$, $\mathrm{y}$, por tanto, con un incremento de tareas, particularmente para las mujeres, es decir, quienes trabajan en una ocupación remunerada fuera del hogar no necesariamente reducen sus actividades domésticas ${ }^{11}$, independientemente de que laboren en la industria $^{12}$ o en el sector educativo, como es el caso de los resultados obtenidos en esta investigación e identificados también en Académicas de posgrado del sector agrícola. ${ }^{13}$ Es decir, esta situación se reproduce también en aquellas mujeres que tienen mayor formación académica e incluso que llegan a tener las mismas oportunidades de desarrollo laboral y de ingresos salariales que el género masculino en determinados niveles, como es el caso del trabajo universitario.

En el análisis del trabajo y la salud desde la visión de género, destaca la inequidad que se establecen entre mujeres y hombres, así como el impacto que ejerce sobre la vida de las personas, evidenciándose, entre otras, el uso diferenciado del tiempo libre pero también una distribución diferencial de la salud. ${ }^{14}$ Estas inequidades son producto histórico de la división sexual del trabajo, que ha llevado a que las mujeres hayan sido infravaloradas, estigmatizadas y que tengan menores oportunidades de desarrollo profesional.

La población femenina y masculina de este estudio no tiene el problema de inestabilidad laboral, ya que quienes laboran en esa universidad cuentan con un contrato colectivo que garantiza la estabilidad y seguridad en el empleo. Los salarios, aunque fijados bilateralmente con el sindicato, son precarios e insuficientes, aunque existe un sistema que permite mejorar el nivel de ingresos a través de un programa de incentivos (becas y estímulos), previa cuantificación de la productividad (docencia, conferencias, investigación, publicaciones, etc.). Alcanzar los estímulos implica incrementar el ritmo y el tiempo del trabajo. Este conjunto de condiciones -que apenas sí dan cuenta de la diversidad de condiciones que deben asumirse como consecuencia de esta modalidad de trabajo- permiten explicar algunos de los resultados presentados en este artículo. Específicamente los relacionados con la distribución diferencial de la salud y con el uso del tiempo libre, el cual se ha minimizado al prolongarse la jornada de trabajo más allá de la estrictamente laboral (“oficial”), o porque el agobio no permite desarrollar - por lo menos en un 
grupo numeroso- diversas actividades contenidas en el tiempo de ocio.

Bajo estos condicionantes se consideró como objetivo de este trabajo analizar la asociación que se establece entre diversas actividades laborales, domésticas y algunas de las realizadas en el tiempo libre, con el género y sus efectos en la salud física y mental en docentes de una universidad pública.

\section{MATERIAL Y MÉTODO}

Se realizó un estudio observacional, transversal y analítico con docentes de una universidad pública, habiéndose calculado una muestra representativa aleatoria mediante un muestreo estratificado con un 10\% de reemplazo y con una fiabilidad del 95\%, dando un total de 294 trabajadores, de los cuales participaron el 68\% (199). Se definieron criterios de inclusión, de exclusión y se solicitó el consentimiento informado. Se aplicó una encuesta de uso epidemiológico, sobre la cual se realizó un estudio piloto. Con este instrumento se recabó información de variables demográficas, sobre condiciones de vida y trabajo doméstico.

Se indagaron exigencias laborales como: extensión de la jornada, trabajo en días de descanso, cuota de producción, tipo de supervisión, peligrosidad en el trabajo, órdenes confusas, trabajo que ocasiona daños a la salud, adopción de posiciones/posturas incómodas o forzadas, hombros tensos, tensión de las muñecas para trabajar, entre otras. Asimismo, si se contaba o no con los estímulos económicos. Dichas variables se analizaron posteriormente por género para observar la posible existencia de una distribución diferencial en la exposición a las exigencias del trabajo.

Entre los daños a la salud -recabados por encuesta- se encuentra la fatiga neurovisual, trastornos del sueño, cefalea tensional, migraña, trastornos cardiocirculatorios, digestivos, músculoesqueléticos, lumbalgia, distrés, depresión, ansiedad, disfonía y fatiga. Se conformaron diagnósticos presuntivos con base en baterias validadas y en los criterios contenidos en el "Instructivo para la recolección, codificación y captura de la encuesta individual para la evaluación de la salud de los trabajadores". ${ }^{15}$

Para evaluar ciertos trastornos en la salud como el distrés, la ansiedad y la depresión, se aplicó el DASS-21, el cual fue validado en población latina. ${ }^{16}$ La escala total del DASS-21 tuvo un coeficiente alfa de 0,96 . Los coeficientes alfa de las subescalas también fueron altos (_ de depresión $=0,93 ;$ _ de ansiedad $=0,86$; _ de estrés=0,91).

En este caso en particular se obtuvo un punto de corte para conformar el diagnóstico de distrés, ansiedad y depresión, tomando en consideración la mediana, la cual fue calculada sumando las posibles respuestas dadas a la escala tipo Likert (1, 2 y 3). La mediana para distrés fue 2 , para ansiedad fue 2 y para depresión se tomó el tercer cuartil (75\%) ya que la mediana puntuaba en cero. El punto de corte también fue de 2 en este caso. Se controlaron variables confundidoras tales como la edad y género cuando fue necesario. La asociación estadística se observó con la Ji Cuadrada y se utilizó el programa JMP8. (SAS Institute, Inc.).

En un segundo momento y dependiendo de los hallazgos, se realizaron análisis estadísticos bivariados para buscar asociaciones y multivariados para identificar diversos efectos que pueden presentarse cuando se combinan diversas variables. En este caso se hizo también un análisis por conglomerados.

\section{RESULTADOS}

Participaron 115 hombres (58\%) y 84 mujeres (42\%), con un promedio de edad de 54,4 años (D.S. de 8,4). El 83\% tiene el grado de maestría y/o doctorado. El 91\% son titulares y de éstos el 85\% tienen la categoría "C" que es la más alta. Una cuarta parte de la población pertenece al Sistema Nacional de Investigadores (SNI) y la distribución entre géneros es equitativa (51\% hombres vs 49\% mujeres). En relación con la Beca a la Permanencia, la cual se obtiene con productos derivados de la investigación: artículos, capítulos de libro, presentaciones nacionales e internacionales, etc. dos terceras partes cuentan con ella (66\%) y la mitad de esta población lleva más de 11 años disfrutando de la misma.

\section{Sobre la exposición a riesgos y exigencias laborales}

Se llevó a cabo una exploración sobre la exposición a algunos riesgos y exigencias laborales que se perfilan como inherentes a las actividades que cotidianamente realizan los docentes. (Cuadro 1).

\section{Cuadro 1.}

Riesgos y exigencias laborales que presenta el personal académico durante el desarrollo de su jornada de trabajo. UAM-X.

\begin{tabular}{lccc} 
Puesto de trabajo lo obliga a: & N & Sí & $\%$ \\
\hline $\begin{array}{l}\text { Realizar trabajos pendientes en dia de descanso } \\
\text { Cubrir determinado número de cursos, clases, }\end{array}$ & 197 & 172 & 87 \\
artículos y conferencias & 198 & 167 & 84 \\
Realizar una tarea demasiado minuciosa & 195 & 121 & 62 \\
Una jornada semanal mayor de 48 hrs. & 197 & 101 & 51 \\
Exposición excesiva a ruido & 193 & 55 & 28 \\
El trabajo le puede ocasionar un daño a la salud & 197 & 42 & 21 \\
Adoptar posiciones incómodas & 198 & 38 & 19 \\
No poder desatender su tarea por más de 5 min. & 195 & 24 & 12 \\
Soportar una supervisión estricta & 194 & 20 & 10 \\
Recibir órdenes confusas de su jefe & 199 & 18 & 9
\end{tabular}

Fuente: Encuesta individual-2009.

En el Cuadro 1 se muestran diversas exigencias laborales a las que se someten los trabajadores en sus tareas diarias. Se observan principalmente actividades que implican la sobrecarga de trabajo como: realizar trabajos pendientes en días de descanso, extensión laboral de más de 48 horas a la semana y cubrir lo que en el sector industrial se denomina "cuota de producción" a través de la preparación e impartición de las clases, elaboración de artículos, conferencias, etc.

Asimismo, una quinta parte de esta población percibe que posiblemente el trabajo le pudiera ocasionar daño/s a su salud. La falta de claridad en el trabajo, valorada a través de la impartición de órdenes confusas, las refieren un porcentaje pequeño de trabajadores.

\section{El perfil de dańo}

En esta investigación se indagó sobre aquellos trastornos en la salud que podrían estar presentando los trabajadores en relación con las actividades ligadas a la docencia y a la investigación. Todos ellos son trastornos crónicos que pueden estar asociados con el contenido cualitativo y cuantitativo de las tareas desarrolladas. El análisis específico del tipo de padecimientos presentado (véase Cuadro 2) muestra al distrés ocupando el primer lugar del perfil de daño. 41 de cada 100 académicos está presentando un conjunto de sintomas que están generando mucho malestar. La disfonía y los trastornos músculo-esqueléticos se están presentando también en casi 4 de cada 10 docentes. Particularmente la disfonía se asoció 
con el tiempo en que se usa la voz de manera cotidiana y el riesgo fue de dos veces más alto en quienes usan la voz de manera continua más de 7 horas al día, en relación con aquellos que la usan hasta tres horas al día. El valor de $\mathrm{p}$ fue $<0,04$. El $30 \%$ señaló que ha percibido alteraciones en el timbre de su voz y el 94\% no ha tenido entrenamiento en el uso de la voz.

Los trastornos del sueño, la ansiedad y la fatiga están presentes en un tercio de la población y es muy probable que guarden correspondencia con el distrés referido por un porcentaje alto de estos trabajadores. Concretamente, la fatiga patológica ha sido estudiada en el sector académico universitario a través de una de las dimensiones del llamado síndrome del estar quemado por el trabajo (burnout): el agotamiento emocional. En el presente trabajo se indagó sobre la fatiga física y mental que pudiera estar asociada con las condiciones de trabajo. La tasa obtenida fue de 3 de cada 10.

\section{Cuadro 2.}

Perfil de daño de los trabajadores académicos de la UAM-X.

$\begin{array}{llcr}\text { Trastornos padecidos durante el último año } & \text { N } & \text { Sí } & \text { Tasa* }^{*} \\ \text { Distrés } & 192 & 79 & 41 \\ \text { Disfonía } & 198 & 76 & 38 \\ \text { Músculo-esquelético } & 198 & 75 & 38 \\ \text { Trastornos del sueño } & 198 & 63 & 32 \\ \text { Ansiedad } & 195 & 62 & 32 \\ \text { Fatiga } & 198 & 57 & 29 \\ \text { Lumbalgia } & 197 & 52 & 26 \\ \text { Fatiga neurovisual } & 195 & 48 & 25 \\ \text { Cefalea tensional } & 196 & 47 & 24 \\ \text { Migraña } & 177 & 40 & 23 \\ \text { Depresión } & 192 & 36 & 19 \\ \text { Psicosomáticos digestivos } & 198 & 19 & 10 \\ \text { Psicosomáticos cardiocirculatorios } & 194 & 8 & 4 \\ \text { TOTAL } & 199 & 662 & 332,6\end{array}$

* Por 100 trabajadores. Fuente: Encuesta individual-2009.

La lumbalgia (tasa de 26 por cada 100), al igual que los trastornos músculo-esqueléticos (38 por cada 100), guarda relación con el trabajo sedentario que es característico de las tareas que desarrolla esta población.

\section{Algunos tópicos relacionados con la vida en el tiempo libre y la distribución por género de tareas diversas}

Se indagó también sobre la realización de diversas actividades domésticas, en tanto llegan a ocupar un lugar importante en la gestión diaria de la vida cotidiana: el 83\% realiza trámites/gestiones de la casa y familia, 81\% hace la compra familiar y 76\% arreglos de la casa, como los porcentajes más elevados. Del conjunto de tareas domésticas estudiadas, mostraron asociación estadística, hacer compras, lavar y planchar ropa, cuidado de hijos y la preparación de la comida, siendo asumidas principalmente por el género femenino.

Cuadro 3.

Actividades domésticas realizadas por la población académica. UAM-X.

$\begin{array}{lcc}\text { Trabajo doméstico } & \text { Si }(\mathrm{N}=199) & \% \\ \text { Realizar trámites de la casa y familia } & 163 & 83 \\ \text { Hacer compras } & 160 & 81 \\ \text { Arreglos en casa } & 149 & 76 \\ \text { Lavar trastos y limpiar la cocina } & 86 & 45 \\ \text { Preparar comida } & 75 & 39 \\ \text { Cuidar hijos: comida, tareas (si tiene hijos) } & 62 & 39 \\ \text { Sacudir, barrer, trapear, hacer camas } & 56 & 30 \\ \text { Lavar y planchar ropa } & 51 & 27 \\ \text { Coser, remendar, zurcir, tejer } & 44 & 24\end{array}$

Fuente: Encuesta individual-2009.
Si bien en esta investigación se optó por no preguntar de manera directa sobre el nivel de ingresos, sí fue posible conocer que para el 87\% el salario le alcanzaba para cubrir sus gastos más necesarios; además de contar con ingresos adicionales como los aportados por su pareja (83\%), el 79\% dispone de vivienda propia, señalando la mitad que en los últimos años habian mejorado las condiciones de la misma.

Se averiguó la distribución de estas tareas por género, resultando que también en esta población las mujeres continúan asumiendo principalmente las tareas domésticas, aunque los hombres se pronunciaron mayormente por los arreglos de casa y lavar la loza. Fuera de estas actividades, así como de los trámites y gestiones del hogar que arrojaron porcentajes similares, el resto de tareas es asumido por las mujeres, todas ellas con diferencias estadísticamente significativas. (Cuadro 4).

\section{Cuadro 4.}

Distribución por género en la realización de actividades domésticas. UAM-X.

\begin{tabular}{|c|c|c|c|c|c|}
\hline Trabajo doméstico & Fem $(1)^{*}$ & Masc (2) & $\mathrm{RP}^{* *}$ & $(\mathrm{I} . \mathrm{C} \text { al } 95 \%)^{* * *}$ & $\mathrm{Ji}^{* * * *}$ \\
\hline Hacer compras & 92 & 73 & 1,3 & $1,11-1,43$ & 0,0005 \\
\hline Trámites de casa & 83 & 83 & 1,0 & $0,88-1,14$ & 0,969 \\
\hline Arreglos en casa & 71 & 80 & 0,89 & $0,76-1,05$ & 0,671 \\
\hline Lavar trastos & 43 & 46 & 0,93 & $0,68-1,29$ & 0,783 \\
\hline Lavar y planchar ropa & 36 & 20 & 1,8 & $1,13-2,94$ & 0,007 \\
\hline Cuidar hijos & 52 & 31 & 1,7 & $1,16-2,49$ & 0,01 \\
\hline Preparar comida & 48 & 33 & 1,5 & $1,04-2,09$ & 0,030 \\
\hline Coser, remendar, zurcir & 38 & 13 & 2,9 & $1,66-5,11$ & 0,0001 \\
\hline Sacudir & 31 & 29 & 1,1 & $0,70-1,69$ & 0,61 \\
\hline
\end{tabular}

*Tasa por 100 trabajadores. **RP Razón de Prevalencia. ${ }^{* *}$ Intervalo con un 95\% de Confianza. ${ }^{* * *}$ Ji ajustada por edad. Fuente: Encuesta individual-2009.

Algo que ha llamado la atención es la homogeneidad en la exposición laboral referida tanto por hombres como por mujeres. De las múltiples exigencias indagadas solamente se encontró asociación en las contenidas en el Cuadro 5. Son las mujeres quienes señalan mayormente la necesidad de realizar trabajos pendientes en horas o días de descanso y de vacaciones; también son ellas quienes perciben la posibilidad de que el trabajo pueda ocasionarles algún daño a su salud. El resto de exigencias de carácter ergonómico fueron también reportadas principalmente por las mujeres alcanzando un riesgo duplicado en el caso de la tensión en hombros con valor de $\mathrm{p}$ estadísticamente significativo.

En relación con el perfil de daño por género, el Cuadro 6 muestra una razón de prevalencia general de 1,47 y en el interior del

\section{Cuadro 5.}

Distribución por género en la exposición a exigencias laborales en académicos universitarios. UAM-X.

\begin{tabular}{|c|c|c|c|c|c|}
\hline EXIGENCIAS & Fem* & Masc & $\mathrm{RP}^{* *}$ & $(\text { I.C a } 95 \%)^{* * *}$ & $\mathrm{Ji}^{* * * *}$ \\
\hline $\begin{array}{l}\text { Realizar trabajos pendientes } \\
\text { en horas o dias de descanso } \\
\text { o vacaciones }\end{array}$ & & & & & 0011 \\
\hline $\begin{array}{l}\text { Al realizar su trabajo los } \\
\text { hombros están tensos }\end{array}$ & 56 & 26 & 2,15 & $1,51-3,16$ & 0,001 \\
\hline $\begin{array}{l}\text { Tuerce o mantiene tensa la o } \\
\text { las muñecas para trabajar } \\
\text { Adoptar posiciones incómodas }\end{array}$ & 34 & 20 & 1,7 & $1,05-2,72$ & 0,028 \\
\hline $\begin{array}{l}\text { o forzadas } \\
\text { Realizar un trabajo que le puede }\end{array}$ & 27 & 14 & 1,92 & $1,07-3,40$ & 0,026 \\
\hline ocasionar algún daño a su salud & 28 & 17 & 1,64 & $0,97-2,85$ & 0,062 \\
\hline
\end{tabular}

*Tasa por 100 trabajadores. **RP Razón de Prevalencia Fem/Masc.

*** Intervalo con un 95\% de Confianza. ${ }^{* * *}$ Ji ajustada por edad. Fuente: Encuesta individual-2009. 
Cuadro 6.

Perfil de daño por género, en docentes universitarios. UAM-X.

$\begin{array}{lccccc}\text { Diagnóstico } & \text { Mujeres* } & \text { Hombres } & \text { R.P** } & \text { (I.C. al 95\%)*** } & \text { Ji**** } \\ \text { Trastornos músculo-esqueléticos } & 51 & 29 & 1,75 & 1,32-2.52 & 0,002 \\ \text { Distrés } & 50 & 35 & 1,42 & 1,03-2,01 & 0,02 \\ \text { Disfonia } & 48 & 31 & 1,54 & 1,08-2,19 & 0,02 \\ \text { Trastornos del sueño } & 41 & 25 & 1,60 & 1,08-2,44 & 0,01 \\ \text { Ansiedad } & 40 & 26 & 1,50 & 1,04-2,36 & 0,02 \\ \text { Fatiga } & 37 & 23 & 1,60 & 1,07-2,56 & 0,02 \\ \text { Cefalea } & 30 & 20 & 1,50 & 0,90-2,43 & 0,10 \\ \text { Fatiga neurovisual } & 29 & 21 & 1,38 & 0,89-2,25 & 0,19 \\ \text { Lumbalgia } & 28 & 25 & 1,12 & 0,70-1,78 & 0,82 \\ \text { Migraña } & 24 & 22 & 1,14 & 0,64-1,91 & 0,62 \\ \text { Depresión } & 22 & 17 & 1,31 & 0,69-2,25 & 0,40 \\ \text { Psicosomáticos digestivos (gastritis) } & 12 & 8 & 1,50 & 0,65-3,62 & 0,33 \\ \text { Psicosomáticos cardio-circulatorios } & 6 & 3 & 2,20 & 0,57-9,45 & 0,22 \\ \text { TOTAL } & 418 & 284 & 1.47 & & \end{array}$

*Tasa por 100 trabajadores. **RP. Razón de prevalencia. ${ }^{* * *}$ Intervalo con un 95\% de Confianza. ${ }^{* * *}$ Ji ajustada por edad. Fuente: Encuesta individual-2009.

perfil se encuentran los trastornos músculo-esqueléticos con un riesgo del 75\% mayor para las mujeres. Se buscó la asociación de este trastorno con las exigencias y el mantener los hombros tensos, se perfiló como la más importante con una $\mathrm{p}<0,0002$, siendo muy significativa. Asimismo, el distrés, la disfonía, los trastornos del sueño, la ansiedad y la fatiga se presentan principalmente en las mujeres con significancia estadística. El resto de trastornos, aunque no alcanza a estar asociado estadísticamente, continúa presentando prevalencias más altas para las mujeres.

En relación con el uso del tiempo libre, los académicos señalaron, como se puede observar en el Cuadro 7, que el 94\% estudia diariamente (por lo menos 5 horas en promedio a la semana), condición que guarda una correspondencia estrecha con su actividad laboral, dado que la actualización permanente es una condición obligada en la vida universitaria. Sin embargo, un porcentaje también alto lee diariamente el periódico, revistas o libros, y salen de paseo. Asimismo, la práctica de algún deporte es realizada, al menos, por 4 de cada 10, condición que se perfila como protectora para la aparición de daños y el mantenimiento de la salud.

\section{Cuadro 7.}

Trabajadores académicos que manifestaron realizar actividades diversas en el tiempo libre y de manera cotidiana. UAM-X.

\begin{tabular}{lccc}
\hline Actividades que realiza & N & Si & $\%$ \\
\hline Estudia con regularidad (más de 5 hrs. a la semana) & 199 & 187 & 94 \\
Lee diariamente el periódico, revistas, libros. & 197 & 174 & 88 \\
Sale a pasear con pareja, hijos, etc. & 197 & 146 & 74 \\
Se reúne frecuentemente con amigos & 198 & 91 & 46 \\
Practica algún deporte (3 o más veces/semana) & 198 & 83 & 42 \\
Actividades comunitarias & 195 & 70 & 36 \\
Ve TV la mayor parte del tiempo libre & 196 & 43 & 22
\end{tabular}

Fuente: Encuesta individual-2009.

Cuando se analizan estas actividades por género, son las mujeres quienes principalmente las están desarrollando; sin embargo, dos actividades alcanzaron significación estadística: la reunión con amigos (55\% mujeres vs 39\% hombres con una $\mathrm{p}<0,02)$ y el desarrollo de actividades comunitarias (45\% vs $29 \%$ respectivamente con una $\mathrm{p}=<0,02$ ).

Resulta interesante conocer el efecto sobre la salud que la ausencia de estas actividades puede tener sobre quienes se manifestaron negativamente sobre el desarrollo de las mismas. Del total de activi-
Cuadro 8.

Asociación entre la falta de ejercicio y daños a la salud en docentes universitarios. UAM-X.

\begin{tabular}{|lcccccc|}
\hline TRASTORNOS & N & NO* & Si & R.P** & (I.C. AL 95\%)*** J|*** \\
\hline Fatiga neurovisual & 194 & 30 & 17 & 1.76 & $1,01-3,08$ & 0,037 \\
Migraña & 176 & 27 & 15 & 1.8 & $0,96-3,39$ & 0,11 \\
Psico-Digestivo & 197 & 13 & 5 & 2.6 & $0,94-7,93$ & 0,054 \\
Distrés & 191 & 47 & 32 & 1.46 & $1,02-2,18$ & 0,01
\end{tabular}

*Tasa por 100 trabajadores. **RP. Razón de Prevalencia. ${ }^{* *}$ Intervalo con un 95\% de Confianza. ${ }^{* * *}$ Ji ajustada por género y edad. Fuente: Encuesta individual-2009.

dades extralaborales estudiadas, hay dos que presentaron mayor asociación con los daños a la salud, una de ellas, la ausencia de una práctica regular del deporte se perfiló como condicionante en la aparición de la fatiga neurovisual, de los psicosomáticos digestivos y el distrés y marginalmente en el caso de la migraña (Cuadro 8).

Por otro lado, diversos docentes practicaban actividades extralaborales de carácter comunitario, político o de servicios. Aunque se exploró esta condición como un indicador del uso del tiempo libre de manera activa y en cierta manera protectora para la salud ${ }^{17}$, se halló que quienes desarrollaban actividades adicionales presentaban mayores trastornos en su salud, perfilándose como una carga más, asociándose principalmente con la lumbalgia y la fatiga, trastornos que presentaron asociación estadística, y con el resto de manera marginal. Dicha condición fue independiente del placer o gusto con que se pudieran desarrollar estas actividades extralaborales (Cuadro 9). Asimismo, son las mujeres quienes principalmente ejercian estas actividades $(\mathrm{p}<0,02)$.

\section{Cuadro 9.}

Asociación entre la realización de actividades comunitarias, políticas o de servicio y daños a la salud. UAM-X.

$\begin{array}{lcccccc}\text { TRASTORNOS } & \text { N } & \text { Si }^{*} & \text { NO } & \text { R.P** } & \text { (I.C AL 95\%) } & \text { *** J J*** } \\ \text { Fatiga } & 194 & 40 & 23 & 1,73 & 1,15-2,73 & 0,036 \\ \text { Lumbalgia } & 193 & 36 & 21 & 1,71 & 1,69-2,69 & 0,027 \\ \text { Fatiga neurovisual } & 191 & 33 & 20 & 1,65 & 1,01-2,71 & 0,06 \\ \text { Trastornos del sueño } & 194 & 41 & 26 & 1,57 & 1,07-2,41 & 0,07 \\ \text { Músculo-esqueléticos } & 194 & 47 & 32 & 1,46 & 1,02-2,09 & 0,08\end{array}$

*Tasa por 100 trabajadores. **RP. Razón de Prevalencia. ${ }^{* * *}$ Intervalo con un 95\% de Confianza *** Ji ajustada por género y edad. Fuente: Encuesta individual-2009.

\section{Análisis por conglomerados}

El análisis por conglomerados (cluster) es una aproximación multivariada para identificar la manera en que se puede asociar la presencia de distintas condiciones laborales con diferentes trastornos. Es una manera de acercarse a la multiexposición, que es el caso de esta población. La aproximación multivariada permite incorporar y controlar el peso que ciertas variables pudieran tener sobre el efecto buscado. En este caso en particular confluyen múltiples variables en la predicción de un conglomerado de efectos o sobre un efecto en particular.

En la Gráfica 1 se muestra la presencia de cuatro conglomerados (conjuntos de trabajadores que presentaron o no las variables en estudio). En este caso en particular se muestra el comportamiento de las siguientes variables de interés y que guardan correspondencia con el uso del tiempo libre y con el consumo de tabaco, es decir, usan activamente su tiempo libre o no y consumen tabaco o no. Posteriormente se cruzaron estas variables con conglomerados conformados con diversos trastornos como: la ansiedad, la depresión, la fatiga y el distrés. La Gráfica 1 muestra 
que el primer conglomerado se caracteriza por un conjunto de trabajadores que en general están realizando diversas actividades por arriba de la media y sólo ver televisión y su experiencia como población fumadora se encuentran por debajo de la media. El tercer conglomerado está caracterizado por un grupo de trabajadores que se encuentra por debajo de la media en relación con el desarrollo de alguna actividad extralaboral. En el cuarto conglomerado, la actividad que predomina en ese grupo de trabajadores es el desarrollo de ejercicio por lo menos tres veces a la semana.

\section{Gráfica 1.}

Conglomerados conformados en función de actividades realizadas durante el tiempo libre y el consumo o no de tabaco, UAM-X 2009.

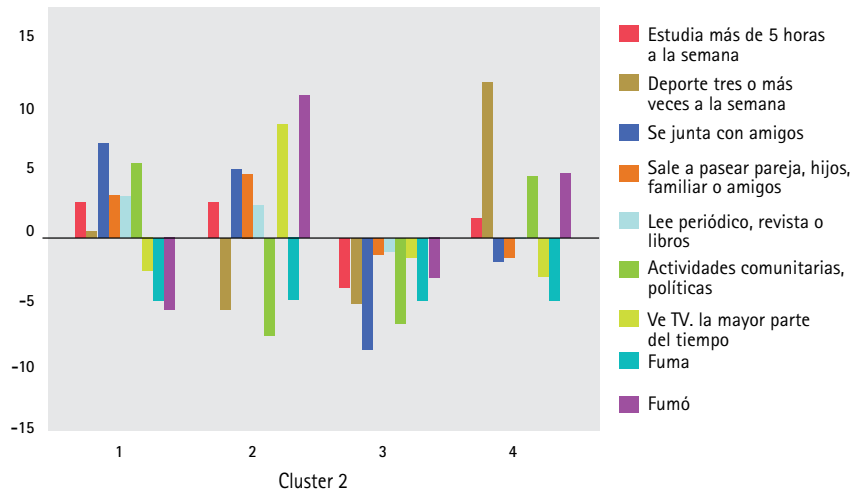

El conglomerado dos está caracterizado por un grupo de trabajadores que fumó en algún momento de su vida y que ve televisión la mayor parte del tiempo libre.

La Gráfica 2 reporta 4 conglomerados relacionados con diversos trastornos. El número 1 corresponde a un grupo de trabajadores que no reportó ninguno de los trastornos en estudio; sin embargo, en el conglomerado cuatro se ubican todos los trabajadores que presentan los cuatro trastornos.

\section{Gráfica 2.}

Conglomerados conformados en función de la presencia o no de distintos trastornos en personal académico, UAM-X 2009.

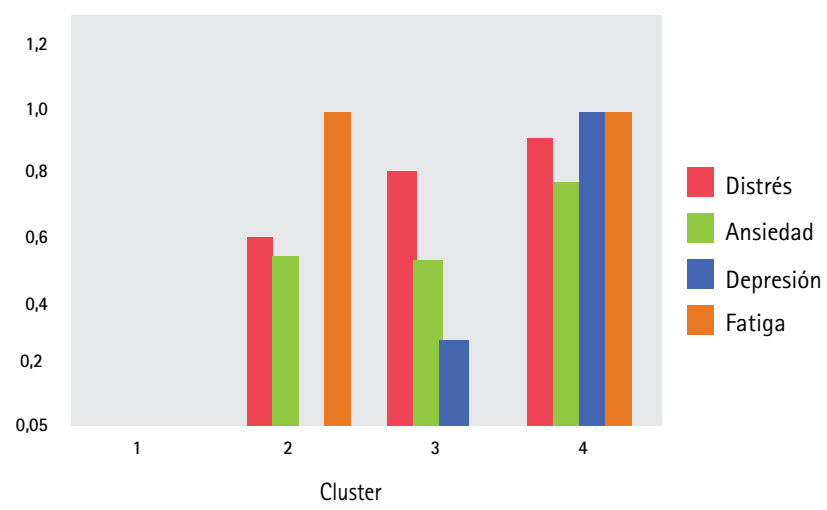

En el análisis de correspondencia (Gráfica 3) se identifican distintos números y colores. Los números señalados con una cruz color rojo (x) son los conglomerados relacionados con diversos trastornos (ansiedad, depresión, distrés y fatiga). Mientras que los señalados con un cuadro color azul son los conglomerados relacionados con uso del tiempo libre (en ambos casos se calcularon 4).
Gráfica 3.

Análisis de correspondencia entre conglomerados. Cuadro azul corresponde a variables de uso de tiempo libre. Cruz roja corresponde a conglomerados de conjunto de daños.

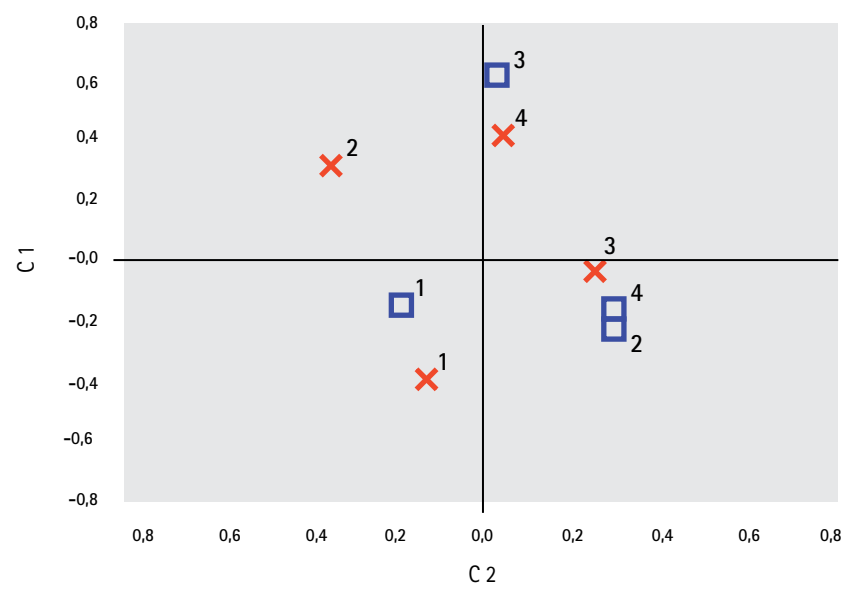

La gráfica muestra la manera en que se relacionan ambos conjuntos de conglomerados. Como se puede observar el conglomerado tres - relacionado con el desarrollo de actividades extralaborales por debajo de la media-, se relaciona de manera estrecha con la mayor presencia de trastornos en la salud: la ansiedad, la depresión, la fatiga y el distrés. Es decir, quienes tienen menor cantidad de actividades extralaborales están presentando una mayor cantidad de trastornos. El nivel de asociación estadística calculada con ji cuadrada es muy alto, la p es $<0,002$. Por otro lado, es posible observar que quienes desarrollan de manera sostenida actividades en el tiempo libre, no presentan ninguno de los trastornos en estudio, lo cual se evidencia con la cercanía que guardan los conglomerados marcados con el número 1 , en esta gráfica.

\section{DISCUSIÓN}

Uno de los planteamientos centrales en el campo de la salud en el trabajo es el nexo que se establece entre la vida laboral y la vida extralaboral, la que se desarrolla en el entorno personal, es decir, en el hogar, en el tiempo libre, en fines de semana y periodos vacacionales. Sin lugar a dudas este espacio reproductivo se ve definido en gran medida por el ingreso salarial que impacta el nivel de satisfactores básicos y ampliados.

Obtener un salario suficiente para cubrir las necesidades básicas implica para esta población participar en un programa de productividad que les permita alcanzar los estímulos económicos. Esto ha llevado a asumir una carga mayor de trabajo y consecuentemente a prolongar la jornada laboral, con menoscabo en el tiempo destinado para la recuperación física y mental como se evidenció en este trabajo.

Respecto a las actividades domésticas, independientemente de que se cuente con el apoyo de las empleadas o asistentes del hogar, diversas tareas son asumidas por esta población y, como se pudo observar, principalmente por las mujeres. La literatura científica reporta que los patrones asignados socialmente a la mujer tienden a reproducirse a lo largo de la vida, motivo por el cual la asunción de labores domésticas forma parte de las tareas cotidianas que 
desarrollan las mujeres y que han pasado a conformar parte de la llamada doble jornada, que igualmente es asumida por los varones de esta población, pero en porcentajes menores. Por lo que se puede señalar que la asunción diferencial de las actividades domésticas por género se perfila como una de las conclusiones más relevantes. Dichas diferencias se mantienen independientemente de que las mujeres que las están asumiendo sean principalmente trabajadoras universitarias, siendo similar a resultados obtenidos en otras universidades. ${ }^{13}$

Por su parte, Giacone y Silva ${ }^{18}$ reportan diversas condiciones que caracterizan el trabajo de las docentes universitarias, algunas de las cuales se reproducen en este estudio, tales como la conjunción del trabajo en casa y el trabajo docente, los tiempos prolongados y las posturas adoptadas durante el trabajo que dañan la columna y articulaciones, las posturas frente a las pantallas electrónicas, la repetición de clases, los cientos de evaluaciones y la conducta no siempre flexible de directivos, titulares y compañeros. Señalan algunas otras características tales como la falta de posibilidades de plantear iniciativas en el trabajo, la subvaloración de ellas como mujeres. Este fenómeno no se exploró en este estudio como tal; sin embargo, en esta universidad mexicana, las oportunidades de que las mujeres asuman puestos de dirección han sido constantes tanto en su calidad de jefas de Área de Investigación, de Departamento, de División Académica e incluso hubo una rectora de Unidad.

En esta investigación también se presenta lo que la literatura científica reporta sobre los daños por género, puesto que generalmente son las mujeres quienes manifiestan una mayor cantidad de sintomas de trastornos a la salud y utilización de los servicios sanitarios. ${ }^{14}$ Respecto a los daños a la salud en este estudio se encontraron hasta 4 trastornos por cada mujer (418 por cada 100 académicas) y poco menos de 3 para los varones (tasa de 284 por cada 100 docentes). La razón de prevalencia de 1,47 muestra un porcentaje más elevado de morbilidad para las mujeres. Fenómeno compartido con lo señalado por Rosales ${ }^{19}$ en España, quien considera que la situación desigual y compleja entre mujeres y varones se relaciona de manera muy clara con sus problemas de salud. Los datos muestran que hay mayor morbilidad en general en las mujeres: dos enfermedades en promedio para ellas por una $\mathrm{y}$ media para los varones.

La Agencia Europea para la Seguridad y la Salud en el Trabajo reportó en 2011 que las mujeres de la región (excluyendo a Francia) presentaron un 60\% problemas músculo-esqueléticos. Los datos de este estudio arrojan al 51\% de mujeres con trastornos de esta naturaleza, porcentaje ligeramente menor. Sin embargo, la ansiedad y la depresión presentaron el 40 y el 22\%, respectivamente, datos mayores si los comparamos con el 16\% reportado por dicha agencia para ambos trastornos. ${ }^{20}$ En un estudio realizado en el Estado de México, la depresión fue mayor en académicas universitarias que en hombres. ${ }^{21}$

También se estudió la manera en que esta población usa su tiempo libre, dado que hay estudios que señalan que cuando los trabajadores tienen un control amplio sobre sus actividades laborales, se da una mayor tendencia a utilizar de manera activa el tiempo de ocio. ${ }^{17}$ Esta población tiene un control amplio sobre muchas de sus actividades laborales, sin embargo, quienes refieren no tener control del ritmo de trabajo presentan mayores problemas en su salud. La vida extralaboral se perfila también como activa; sin embargo, en este caso llama la atención que quienes tienen mayores compromisos comunitarios, políticos o de servicio presentan mayores trastornos. Estos resultados contradicen a quienes señalan que el uso activo del tiempo libre se constituye como una condición protectora para la salud. No es el caso de esta población, para quienes estas actividades extralaborales se convierten en una carga extra de trabajo.

En relación con el género, la literatura señala que por lo regular los varones disponen de mayor tiempo libre que las mujeres y que el tiempo libre ellas lo destinan generalmente a la realización de quehaceres domésticos y al cuidado de los hijos. Es decir, la mujer cumple socialmente los papeles de madre, esposa y ama de casa, además del de trabajadora, y cada uno de ellos le exige cierto tiempo y la realización de determinadas actividades. Esto no permite una división clara entre tiempo de trabajo y tiempo libre. ${ }^{19}$ Este fenómeno no se reprodujo en esta investigación, ya que son las mujeres quienes principalmente hacen un uso más activo de su tiempo libre, dado que disponen del mismo para reunirse con amigos y para desarrollar actividades comunitarias. Seguramente el nivel académico que han alcanzado les facilita disponer de esta manera de su tiempo libre. Por supuesto, el ingreso salarial es mejor, lo que permite en muchas ocasiones la ayuda de las trabajadoras domésticas.

La ausencia de la práctica regular de deporte se perfiló como condicionante en la aparición de la fatiga neurovisual, sintomatología psicosomática digestiva, distrés y, marginalmente, migraña. Es conocido el peso protector que puede tener el deporte -actividades moderadas y vigorosas- en la prevención del riesgo cardiovascular y también es conocido el papel que el distrés tiene en la aparición de estos trastornos. La ausencia de ejercicio se asoció con distrés, lo que puede sentar las bases para la aparición de trastornos cardiovasculares a mediano plazo.

La Organización Panamericana de la Salud ${ }^{22}$ refiere como actividad recomendada una práctica que sea mayor o igual a tres horas a la semana. El porcentaje de académicos que refirieron desarrollar una actividad recomendada fue menor de 50\%, lo que habla de la importancia de tomar en consideración esta actividad.

\section{CONCLUSIONES}

Lo que una persona realiza en su tiempo libre es una cuestión personal; si bien la empresa/organización debe establecer un marco para la armonización entre la vida laboral con la personal, no debe olvidarse que son los gobiernos de cada nación quienes deben disponer y desarrollar políticas y recursos para hacerlo posible.

La incorporación e incremento de las mujeres en el entorno académico exige la creación de un nuevo modelo de trabajo que permita tanto al hombre como a la mujer poder trabajar una jornada completa y cuidar ambos de su familia, compartiendo responsabilidades por partes iguales; ello debe considerarse teniendo en cuenta las nuevas formas de organización familiar.

La distribución diferencial en el perfil de daño por género es un fenómeno frecuente en los estudios de salud en el trabajo, siendo generalmente las mujeres quienes reportan más daños y mayor utilización de los servicios de salud.

El uso del tiempo libre, por debajo de la media, mostró el peso específico que sobre la salud puede tener esta ausencia.

El contexto global actual del mundo laboral está desembocando en trabajos y trabajadores esclavos del tiempo, de forma perma- 
nente y sin fin; la manera de prevenirlo está en el compromiso, respeto e implicación tanto de la empresa/organización por el tiempo libre como de la propia persona trabajadora; en el presente estudio conlleva que la utilización adecuada del tiempo libre es un amortiguador del estrés laboral y, especialmente con la configuración actual del sistema de incentivos (becas), debe considerarse que participar en este tipo de programas es una elección personal que depende en gran medida de lo que la persona quiera sacrificar y/o renunciar.

Se recomienda una mayor organización de las actividades laborales y extralaborales que permitan un uso más activo del tiempo libre, privilegiando el ejercicio físico, que se perfiló como un elemento protector contra trastornos en la salud física y mental.
1. Acevedo D. Género y flexibilización del trabajo. Impacto en la salud. Cuadernos Mujer Salud. 1999;2:95.

2. Rendón Gan T. Participación femenina en la actividad económica. Doble jornada femenina y bajos salarios. Demos. Carta demográfica sobre México. 2003:16.

3. Gómez $\mathrm{S}$, Martí C. La incorporación de la mujer al mercado laboral: implicaciones personales, familiares y profesionales, y medidas estructurales de conciliación trabajo-familia. Pamplona: Universidad de Navarra-IESE Business School. 2004. (Documento de investigación; 557).

4. Maldonado VM, Rendón T. Vínculos entre trabajo doméstico, segregación ocupacional y diferencias de ingreso por sexo en el México actual. México: Instituto de Estudios del Trabajo; 2004.

5. López F, Carrión MA. Más allá de los programas de ayuda al empleado (PAE). En: Carrión MA, López-Barón F, Tous J. Promoción de la salud ocupacional. Barcelona: AEPA- Asociación de Expertos en Psicosociología Aplicada; 2008. p.215-235. (Colección de psicosociología aplicada a la Salud Ocupacional; 2).

6. Artazcoz L, Moncada S. Organización del trabajo, satisfacción laboral y salud mental en trabajadores. Primer Congreso Internacional Mujeres, trabajo y salud; 1996 abr; Barcelona. Barcelona: 1996.

7. Noriega $M$, Gutiérrez $G$, Méndez I, y Pulido $M$. Las trabajadoras de la salud: vida, trabajo y trastornos mentales. Cad Saúde Pública. 2004;20(5): 1361-1372.

8. Urquidi LA, y Rodriguez JR. Estrés en profesorado universitarios mexicano. Actualidades investigativas en educación. 2010;10(2):1-21.

9. Trilla J. Educación formal, no formal e informal. En: Cliffor MM. Enciclopedia práctica de la pedagogía. Tomo 4. Madrid: Océano-Exito; 1983.

10.- Poch R. Trabajar en fin de semana se convierte en norma. La Vanguardia. 2012; 12 Jul.

11. Acevedo D. Género y flexibilización del trabajo; impacto en la salud. Cuadernos Mujer Salud. 1999;2:28-36.
12. Cruz C, Noriega $M$, Garduño A. Salud y vida cotidiana en el ámbito doméstico en la población trabajadora de una empresa farmacéutica. Salud Trab. 2001; 9(2):53-64.

13. Martínez S, Vázquez I. Programas de estímulos, productividad y salud en académicos de posgrado. Salud Trab. 2001;9(1):5-20.

14. Gómez E. Género equidad y acceso a los servicios de salud: una aproximación empírica. Rev Panam Salud Públ. 2002;11(5/6):327-334.

15. Noriega M, Franco G, Martínez S, Villegas J, Alvear G, López J. Evaluación y Seguimiento de la Salud de los Trabajadores. México: UAM-Xochimilco; 2001. (Académicos CBS; 34).

16. Daza P, Novy D, Stanley M, Averill P. The Depression, Anxiety, Stress Scale-21: Spanish Translation and Validation with a Hispanic Sample. J Psychopathol Behav. 2002;24(3):195-205.

17. Karasek $R$, Theorell $T$. Healthy Work. Stress, productivity, and the reconstruction of working life. New York: Basic Books, Inc.;1990.

18. Giacone S, Silva M. Trabajo y salud de las docentes de la Universidad Nacional de Córdoba: uso de medicamentos/sustancias lícitas y plan materno infantil. Rev Latino-Am Enfermagem. 2004;12(No.esp.):383-390.

19. Rosales M. Trabajo, salud y sexualidad. Las cargas de trabajo laborales y reproductivas en la salud de las mujeres. Barcelona: Icaria; 2002.

20. European Agency for Safety and Health at Work 2012. Annual Report 2011 [on line]. Luxembourg: Office for Official Publications of the European Union; 2012 [consultado 7 jul 2012]. Disponible en: https://osha.europa.eu/en/publicats/ annual_report/2011full

21. Contreras $\mathrm{G}$, Veytia $\mathrm{M}$, Huitrón $\mathrm{G}$. Detección del trastorno depresivo en profesores universitarios. Psicología y salud. 2009;19(1):56-70.

22. Estrategia mundial sobre alimentación saludable, actividad física y salud (DPAS). Organización Panamericana de la Salud. Plan de implementación en América Latina y el Caribe 2006-2007. Washington D.C.:OPS; 2006. 